\title{
Churg-Strauss syndrome from an orthopaedic perspective
}

\author{
KL Kung *, PK Yee
}

\section{A B S T R A C T}

Churg-Strauss syndrome, which has been frequently described by physicians in the literature, is a small and medium-sized vessel systemic vasculitis typically associated with asthma, lung infiltrates, and hypereosinophilia. We report a case of ChurgStrauss syndrome with presenting symptoms of bilateral lower limb weakness and numbness only. The patient was admitted to an orthopaedic ward for management and a final diagnosis was reached following sural nerve biopsy. The patient's symptoms responded promptly to steroid treatment and she was able to walk with a stick 3 weeks following admission. This report emphasises the need to be aware of this syndrome when managing patients with neurological deficit in order to achieve prompt diagnosis and treatment.

\section{Hong Kong Med J 2015;21:565-8 \\ DOI: $10.12809 / \mathrm{hkmj} 144357$}

KL Kung *, MB, BS

PK Yee, MB, ChB, FHKCOS

Department of Orthopaedics and Traumatology, Pamela Youde Nethersole Eastern Hospital, Chai Wan, Hong Kong

* Corresponding author: lepetitcarmen@gmail.com

\section{Introduction}

Churg-Strauss syndrome (CSS) is a small and medium-sized vessel vasculitis that can affect different organs. The usual presentation is suboptimised control of asthma together with involvement of other organs such as the heart, skin, and nervous system. It seldom presents with isolated neurological symptoms and has thus far not been reported from an orthopaedic aspect. We report a case of CSS in a patient who presented with neurological symptoms and who was admitted to the orthopaedic ward via casualty because of lower limb weakness and numbness.

\section{Case report}

A 66-year-old Chinese woman was admitted to the orthopaedic ward for 2 weeks in May 2013 because of weakness and numbness in both lower limbs. She had a more than 10 years' history of asthma that was controlled with an inhaled bronchodilator and oral theophylline and terbutaline. The patient was prescribed an oral steroid intermittently for acute control. From January 2012 until the current admission, she had also been taking a leukotriene receptor antagonist (montelukast).

On admission, the patient complained of severe dysesthesia over both lower limbs, mainly below knee level. There was mild left proximal thigh pain. She had no low back pain and denied a history of recent trauma. The symptoms rendered the patient unable to walk.

Upon physical examination, she was afebrile, and cardiopulmonary and dermatological examination was unremarkable. Neurological examination revealed decreased sensation over both lower limbs in a glove and stocking distribution. Power of the muscles supplied by the peroneal and tibial nerves was grade 4 according to Medical Research Council grading system. Reflexes were diminished over both lower limbs. Per rectal examination revealed normal anal tone.

Blood tests revealed elevated white cell count to $28.07 \times 10^{9} / \mathrm{L}$ with a predominance of eosinophils $\left(20.46 \times 10^{9} / \mathrm{L}\right)$. C-reactive protein was $65 \mathrm{mg} / \mathrm{L}$. Erythrocyte sedimentation rate was $52 \mathrm{~mm} / \mathrm{h}$. Serum calcium, phosphate, alkaline phosphatase and creatine kinase level were normal. Sepsis workup including blood culture and urine culture were negative (Table).

Radiography of the lumbar spine revealed grade-one spondylolisthesis at the lumbar 4 and 5 level. Radiography of the pelvis was unremarkable. Chest radiography showed slightly hyperinflated lung. Subsequent magnetic resonance imaging with contrast of the lumbar spine and pelvis was performed in view of the radiographic findings of the lumbar spine and the neurological deficit in the lower limbs. There was neither evidence of nerve root and cord compression nor infection around the lumbar spine.

Nerve conduction study showed absence of the compound muscle action potential (CMAP) of the right peroneal nerve recorded at the extensor digitorum brevis muscle. The CMAP was also decreased over bilateral tibialis anterior muscles. 


\section{從骨科角度看Churg-Strauss綜合徵 龔錦鈴、俞柏堅}

Churg-Strauss綜合徵在文獻中記載甚多, 這種病主要累及中、小型 血管的系統性血管炎, 病變一般與哮喘、肺浸潤和嗜伊紅細胞增多症 有關。本文報告一名病人病發時只出現雙腿無力和麻痺症狀。她被送 入骨科病房, 經腓腸神經活檢後才被確診為Churg-Strauss綜合徵。 病人接受類固醇治療後病情迅速改善, 入院三個星期後已經可以拄着 柺杖走路。這病例顯示當病人有神經功能缺損時, 醫生應考慮ChurgStrauss綜合徵的可能性, 讓病人得到及時診斷和適當的治療。
These results were suggestive of an axonal type of motor neuropathy over bilateral peroneal nerves and a sensory type of axonal neuropathy over the right lower limb. There was no involvement of the upper limbs.

Left sural nerve biopsy revealed epineurial extravascular eosinophils and vasculitis associated with axonal degeneration (Fig). There was one small epineurial artery infiltrated by eosinophils, polymorphs, and lymphocytes. Vague granuloma were present in the vascular wall. These features were consistent with CSS based on the American College of Rheumatology Classification criteria. ${ }^{1}$ The Table summarises the investigations.

A rheumatologist was consulted who diagnosed CSS with peripheral neuropathy. Montelukast was discontinued in view of the possible association of the CSS. Oral prednisolone $40 \mathrm{mg}$ daily was prescribed to the patient and an oral bisphosphonate was given to prevent osteoporosis. Distal lower limb power improved to grade 5 shortly following steroid treatment and there was marked improvement in pain and numbness. The eosinophil count and elevated inflammatory markers reduced

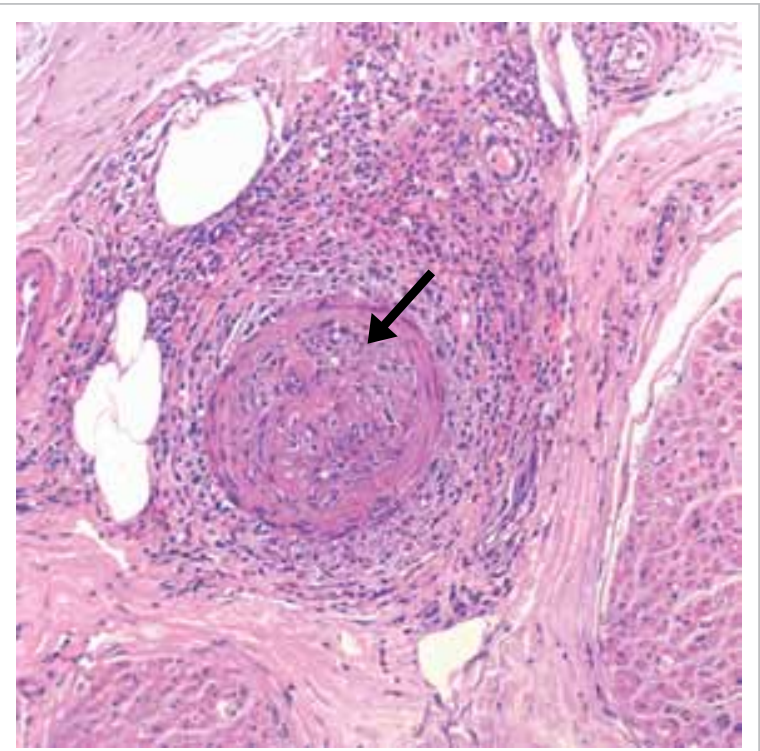

FIG. Microscopy of sural nerve biopsy showing epineurial extravascular eosinophils and vasculitis associated with axonal degeneration; vague granuloma is formed in the vascular wall (arrow) [H\&E; original magnification, $x$ 100]

rapidly over 6 days. The patient was discharged from the orthopaedic ward 2 weeks after treatment and was able to walk with a stick. The patient has been referred to a day hospital for further rehabilitation.

\section{Discussion}

Churg-Strauss syndrome is an entity frequently described by rheumatologists in the literature. ${ }^{2}$ It has seldom been reported by an orthopaedic surgeon. This case illustrates that common symptoms such as pain and numbness, frequently encountered when treating orthopaedic patients, may not be necessarily due to an orthopaedic problem. It may be a medical disease that requires prompt and specific treatment. Allergic granulomatosis angiitis, or CSS, is

TABLE. Summary of investigations

\begin{tabular}{lcc}
\hline Investigations & Reference range/level & Value \\
\hline Total white cell count $\left(\times 10^{9} / \mathrm{L}\right)$ & $3.7-9.7$ & 28.07 \\
Absolute eosinophil count $\left(\times 10^{9} / \mathrm{L}\right)$ & $0.0-0.6$ & 20.46 \\
ESR $(\mathrm{mm} / \mathrm{h})$ & $<20$ & 52 \\
CRP $(\mathrm{mg} / \mathrm{L})$ & $<80$ & 65 \\
ANCA & - & Negative \\
Serum creatinine $(\mu \mathrm{mol} / \mathrm{L})$ & $<110$ & 51 \\
Chest X-ray & - & Hyperinflated lung fields \\
Nerve conduction studies & - & Exonal sensorimotor neuropathy \\
Sural nerve biopsy & - & Eosinophilic vasculitis with axonal degeneration \\
\hline
\end{tabular}

Abbreviations: ANCA = antineutrophil cytoplasmic antibody; CRP = C-reactive protein; ESR = erythrocyte sedimentation rate

* By indirect immunofluorescence assay 
a type of antineutrophil cytoplasmic antibodyassociated small-vessel systemic vasculitis. Other diseases under the same group include microscopic polyangiitis and granulomatosis with polyangiitis, formerly known as Wegener's granulomatosis. ${ }^{3}$ It was first described in 1951 by Dr Jacob Churg and Dr Lotte Strauss at Mount Sinai Hospital and is characterised by eosinophilic vasculitis that affects the small and medium-sized vessels. It describes the clinical symptoms of the pathological entity allergic angiitis and granulomatosis. The American College of Rheumatology has recommended that diagnosis of the syndrome is considered when four of the following features are present: (1) asthma, (2) eosinophils constituting more than $10 \%$ of the white cell count, (3) neuropathy, (4) non-fixed pulmonary infiltrates on radiography, (5) extravascular granulomas, and (6) abnormalities of the paranasal sinuses. ${ }^{1}$ The presence of four or more criteria yields a diagnostic sensitivity of $85 \%$ and a specificity of 99\%. ${ }^{1}$

Despite variable clinical manifestations, pathological findings will include necrotising eosinophilic vasculitis that may result from endothelial cell adhesion and leukocyte activation, with subsequent necrotising vasculitis in several organ systems.

The pathogenesis of the syndrome is unclear, but it has been shown that $H L A-D R B 3$ is a genetic risk factor for development. There have been reports about the strong association between CSS and the use of a leukotriene receptor antagonist (LTRA) such as montelukast. The mechanism by which LTRAs might cause eosinophilic vasculitis remains unclear, however. It is known that LTRAs do not inhibit leukotriene B4 (LTB4), which is a powerful chemoattractant for eosinophils. This could lead to increased plasma levels of LTB4 and trigger eosinophilic inflammation. Nevertheless, LTRA has a somewhat causative role in the development of CSS. A controlled study with a large number of patients is required to verify this conclusion.

The clinical presentation is variable. Some people have only mild symptoms, while others experience severe or life-threatening complications. There are three stages of CSS, but not every patient will develop all three phases or in the same order. They include:

(1) Allergic stage: the first stage of the syndrome in which the patient develops a number of allergic reactions including asthma, hay fever, and sinusitis.

(2) Eosinophilic stage: hypereosinophilia is found in the blood or tissue causing serious local damage. The lungs and digestive tract are most often involved. The symptoms may be relapsing and remitting and last for months or years.
(3) Vasculitic stage: the hallmark of this stage is blood vessel inflammation. The blood vessels are narrowed by inflammation and blood flow to tissue is jeopardised. During this phase, a feeling of general ill-health along with unintended weight loss, lymphadenopathy, weakness, and fatigue may occur.

Asthma is the central feature of CSS and precedes the systemic manifestations in almost all cases. Upper airway findings can include sinusitis, allergic rhinitis, and nasal polyps. Cardiac involvement represents the major cause of mortality. Granulomatous infiltration of the myocardium and coronary vessel vasculitis are the most common lesions. Congestive heart failure may develop rapidly and is often responsible for the mortality. Gastro-intestinal involvement such as abdominal pain, diarrhoea, and bleeding may also occur. Bowel perforation is the most severe manifestation and is one of the major causes of death. The glomerular lesion that typifies CSS is focal segmental glomerulonephritis with necrotising crescents. Its involvement is one of the poor prognostic factors. Arthralgias are frequent but arthritis with local inflammatory findings is rare, and joint deformity and radiographic erosions are usually not seen. Large articular joints are affected more than small joints. The symptoms usually regress quickly with treatment. Skin involvement occurs in 50\% to $68 \%$ of patients and reflects the predilection for small vessels. Lesions are red or violaceous, and occur primarily on the scalp and the limbs or hands and feet. They are often bilateral and symmetrical. ${ }^{4}$

Peripheral neuropathy, as in our case, is common in patients with CSS (65-75\%). ${ }^{5,6}$ The initial symptoms of neuropathy usually include an acute onset of tingling or painful paresthesia in the extremities. These symptoms predominantly occur in the distal portions of the extremities, particularly the lower limbs. In the initial phase, the distribution of sensory impairment usually takes the form of a mononeuritis multiplex pattern. As the disease progresses, it eventually evolves into a polyneuropathic pattern. Muscle weakness, to a variable extent and degree of asymmetry, may also be evident. There will be decreased or absent deep tendon reflexes corresponding to the distribution of nerves involved. Electromyography reveals axonal nerve involvement and often detects more extensive involvement than the clinical symptoms would indicate. With treatment, mononeuritis multiplex regresses progressively and patients may recover without sequelae. Prompt diagnosis and treatment are of paramount importance in managing CSS with neuropathy. If medical treatment is delayed, atrophy and weakness of the limbs may be irreversible and will require additional rehabilitation therapy including strengthening exercises, balance training, 
and ambulation training.

In the treatment of CSS, as with other forms of vasculitis, the symptoms respond to corticosteroids or other immunosuppressive drugs, eg cyclophosphamide and methotrexate. ${ }^{7}$ If refractory to these agents, intravenous immunoglobulin is the treatment of choice. Leukocytosis, eosinophilia, and raised erythrocyte sedimentation rates usually respond promptly after administration of these agents. Neuropathic pain also decreases rapidly in $85 \%$ of patients. There is speculation that the initial clinical course and the degree of systemic inflammatory involvement may influence long-term functional prognosis. Recent trials of treatment include immunomodulators such as rituximab, an anti-CD20 monoclonal antibody, and tumour necrosis factor-alpha. ${ }^{8}$

In this article, we have reviewed the clinical features, diagnostic criteria, and treatment options of CSS. In middle-aged or elderly patients, lower limb numbness and pain are frequently attributed to the degenerative spine with stenosis or nerve root compression. It is tempting to conclude that a patient has spinal stenosis or lumbar spondylosis in the presence of degenerative lumbar spine radiographs. This case highlights the need for clinicians to be vigilant for these manifestations in addition to the various diagnoses of spinal pathology when a patient presents with limb numbness and weakness together with a history of asthma and raised eosinophil count. Because signs and symptoms are both numerous and at times unassuming, it is notoriously difficult to diagnose CSS at the very initial phase. Nonetheless, significant neuropathic involvement may be prevented if a patient receives adequate therapy to induce remission of disease and prevent relapse. With treatment, most of the symptoms in any of the three phases can be relieved. We would like to raise the awareness of such an entity in treating patients with neuropathy. Close collaboration with rheumatologists in treating such a complex illness, including prompt diagnosis by performing nerve biopsy by an orthopaedic surgeon and prompt medical treatment by a rheumatologist, is the key to successful management.

\section{References}

1. Masi AT, Hunder GG, Lie TT, et al. The American College of Rheumatology 1990 criteria for the classification of Churg-Strauss syndrome (allergic granulomatosis and angiitis). Arthritis Rheum 1990;33:1094-100.

2. Churg J, Strauss L. Allergic granulomatosis, allergic rhinitis, and periarthritis nodosa. Am J Pathol 1951;27:277-301.

3. Falk RJ, Gross WL, Guillvein L, et al. Granulomatosis with polyangiitis (Wegener's): An alternative name for Wegener's granulomatosis. Arthritis Rheum 2011;63:8634.

4. Guillevin L, Pagnoux C, Mouthon L. Churg-Strauss syndrome. Semin Respir Crit Care Med 2004;25:535-45.

5. Sehgal M, Swanson JW, DeRemee RA, Colby TV. Neurologic manifestations of Churg-Strauss syndrome. Mayo Clin Proc 1995;70:337-41.

6. Hattori $N$, Ichimura $M$, Nagamatsu $M$, et al. Clinicopathological features of Churg-Strauss syndromeassociated neuropathy. Brain 1999;122:427-39.

7. Bosch X, Guilabert A, Espinosa G, Mirapeix E. Treatment of antineutrophil cytoplasmic antibody associated vasculitis: a systematic review. JAMA 2007;298:655-69.

8. Thiel J, Hässler F, Salzer U, Voll RE, Venhoff N. Rituximab in the treatment of refractory or relapsing eosinophilic granulomatosis with polyangiitis (Churg-Strauss syndrome). Arthritis Res Ther 2013;15:R133. 